\section{Statins for primary prevention}

We wish to address an important issue that relates to the analysis of serious adverse events in the systematic review of statins for primary prevention by Tonelli and colleagues. ${ }^{1}$

In the results section, the authors indicate " $\ldots$ the pooled risk of serious adverse events did not differ significantly between treatment groups (RR $1.01,95 \%$ CI $\left.0.96-1.07 ; I^{2}=8 \%\right)$." Although we congratulate the authors for obtaining data on serious adverse events from 21 of 29 trials, we urge them to post the forest plot and raw data from this analysis on CMAJ's website for use by other researchers. The pooled relative risk confirms the finding of the Therapeutics Initiative of the University of British Columbia, that total serious adverse events are not reduced by statins in the clinical setting of primary prevention. ${ }^{2,3}$ The Therapeutics Initiative concluded that statins do not have a proven net health benefit in primary prevention. The analysis by Tonelli and colleagues, which furnishes data on serious adverse events from more trials, strengthens that conclusion.

What does the estimate of relative risk for serious adverse events reported by Tonelli and colleagues mean in clinical terms? The RR of 1.01 implies that taking a statin increases the chance of dying, being admitted to hospital or being permanently disabled by a nonsignificant $1 \%$ as compared with placebo. A conservative assumption is that serious adverse events in those trials were more frequent in the statin group than in the placebo group.

Why do Tonelli and colleagues ignore the serious adverse events outcome in their conclusions? Perhaps the data create a dilemma for proponents of widespread statin use in primary prevention. How can statins cause a statistically significant reduction in all-cause mortality, nonfatal myocardial infarction and nonfatal stroke - all outcomes that rep- resent serious adverse events and that should be counted as such within the data on serious adverse events reported in clinical trials - and yet not reduce total serious adverse events? This implies that statins increase the risk of other serious adverse events to negate the decreased risk in cardiovascular morbidity and mortality.

In summary, we consider that the most important finding in the metaanalysis by Tonelli and colleagues is that statins do not reduce total serious adverse events and thus do not provide a net health benefit in primary prevention.

\section{Aaron M. Tejani BSc(Pharm) PharmD Vijaya Musini MD MSc \\ Ken Bassett MD PhD \\ Colin Dormuth MSc ScD \\ Tom Perry MD \\ James M. Wright MD PhD \\ Departments of Anesthesiology, \\ Pharmacology \& Therapeutics, University \\ of British Columbia, Vancouver, BC}

\section{References}

1. Tonelli M, Lloyd A, Clement F, et al.; for the Alberta Kidney Disease Network Efficacy of statins for primary prevention in people at low cardiovascular risk: a meta-analysis CMAJ 2011;183: E1189-1202.

2. Do statins have a role in primary prevention? Therapeutics Letter 2003;48:1-2. Available: www.ti.ubc .ca/newsletter/do-statins-have-role-primary-prevention (accessed 2011 Dec. 13 2011).

3. Do statins have a role in primary prevention? An update. Therapeutics Letter 2010;77:1-2. Available: www.ti.ubc.ca/letter77 (accessed 2011 Dec. 12).

\section{CMAJ 2012. DOI:10.1503/cmaj.112-2036}

\section{The authors respond}

Tejani and colleagues ${ }^{1}$ point out that further consideration should be given to the merit of serious adverse events as an overall marker of the benefit of any therapy — including statins.

The letter by Tejani and colleagues, in response to our article, ${ }^{2}$ includes an alternative interpretation of our estimate of the pooled risk of serious adverse events and withdrawals across treatment groups. This interpretation is interesting but is not widely used for several reasons.

First, the definition and reporting of serious adverse events are not typically standardized, nor do they typically include the prespecified primary outcome for the trial. Both of these limitations can introduce error when estimating the effect of treatment on serious adverse events. If the pooled analysis of the primary outcomes of the trial (e.g., nonfatal myocardial infarction or coronary death) suggested no benefit for recipients of statins but showed that serious adverse events were less common in the statin group, would Tejani and colleagues have suggested that we ignored the data if we concluded that statins were ineffective?

Second, the interpretation of Tejani and colleagues assumes that efficacy outcomes, such as myocardial infarction and mortality, are included in the analysis of total serious adverse events observed. Our interpretation is based on a more conventional assumption - that efficacy outcomes are removed from analyses of serious adverse events. We based our assumption on first-hand experience with previous trials as well as on supplementary trial data. For example, the US Food and Drug Administration medical reviewer's report of the JUPITER trial states "Any adjudicated cardiovascular death was classified as an efficacy endpoint and not an [adverse event]." ${ }^{3}$ Despite our best efforts, we could not always determine whether efficacy and safety outcomes were analyzed separately, based on available trial reports. Nonetheless, because we agree that serious adverse events are clinically important, we took extra care to identify and report these analyses in our paper.

Third, our definition of serious adverse events also included events that led to withdrawal of assigned treatment, not only those that were life-threatening. Because not all withdrawals from treatment are clinically relevant, some of the concerns raised by Tejani and colleagues may be less applicable than their letter suggests.

Despite these caveats, we acknowledge that properly interpreting how harm was experienced, measured and 
reported by trialists is of special importance when achievable benefits are low, and that our finding of lower cardiovascular and mortality risk is sensitive to this assumption. We thank Tejani and colleagues for raising this important point.

\section{Donald Husereau BScPharm MSc}

Department of Epidemiology and Community Medicine, University of Ottawa,

Ottawa, Ont.

Braden Manns MD MSc

Departments of Medicine and of Community Health Sciences, University of Calgary, Calgary, Alta., and Alberta Kidney Disease Network, Calgary/Edmonton, Alta.

Anita Lloyd MSc

Marcello Tonelli MD SM

Department of Medicine, University of Alberta, Edmonton, Alta.; and Alberta Kidney Disease Network, Calgary/Edmonton, Alta.

\section{References}

1. Tejani, et al. Statins for primary prevention [letter]. CMAJ 2012;184:791.

2. Tonelli M, Lloyd A, Clement F, et al.; Alberta Kidney Disease Network. Efficacy of statins for primary prevention in people at low cardiovascular risk: a meta-analysis. CMAJ 2011;183:E1189-202.

3. Roberts MD. Application number: 21-366/s-016. Medical review(s). CRESTOR ${ }^{\circledR}$ (rosuvastatin calcium). Silver Spring (MD): Center for Drug Evaluation and Research; 2010. Available: www.accessdata .fda.gov/drugsatfda_docs/nda/2010/021366s016MedR .pdf (accessed 2012 Feb. 8).

CMAJ 2012. DOI:10.1503/cmaj.112-2037

\section{-Correction \\ Article link \\ In the editorial published in the Mar. 6, 2012 issue of $C M A J,{ }^{1}$ the incorrect link was provided to the related News article. The correct link is www.cmaj.ca/lookup/doi /10.1503/cmaj.109-4091. CMAJ regrets the error. \\ Reference \\ 1. Kale R. "It's a girl!" - could be a death sentence. CMAJ 2012;184:387-8. \\ CMAJ 2012. DOI:10.1503/cmaj.112-2039}

Some letters have been abbreviated for print. See www.cmaj.ca for full versions and competing interests. 